\title{
Effectiveness of SIESTA on Objective and Subjective Metrics of Nighttime Hospital Sleep Disruptors
}

Vineet M Arora, MD MAPP*; Nolan Machado, BA²; Samantha L. Anderson, BA; Nimit Desai, MD; William Marsack, MSN; Stephenie Blossomgame, MSN ${ }^{1}$; Ambrosio Tuvilleja, RN¹; Jacqueline Ramos, RN ${ }^{1}$; Mary Ann Francisco, $\mathrm{MSN}^{1}$; Cynthia LaFond, PhD, RN, CCRN³; Edward KY Leung, PhD³; Andres Valencia, BA; Shannon K Martin, MD, MS; David O Meltzer, MD, PhD'; Jeanne M Farnan, MD, MHPE' , Jay Balachandran, MD, Kristen L Knutson, PhD5 ${ }^{5}$, Babak Mokhlesi, MD, MSc

${ }^{1}$ University of Chicago Medicine, Chicago, Illinois; ${ }^{2}$ Pritzker School of Medicine, Chicago, Illinois; ${ }^{3}$ Children's Hospital Los Angeles, Los Angeles, California; ${ }^{4}$ Columbia-St. Mary's, Mequon, Wisconsin; ${ }^{5}$ Northwestern University, Chicago, Illinois.

We created Sleep for Inpatients: Empowering Staff to Act (SIESTA), which combines electronic "nudges" to forgo nocturnal vitals and medications with interprofessional education on improving patient sleep. In one "SIESTAenhanced unit," nurses received coaching and integrated SIESTA into daily huddles; a standard unit did not. Six months pre- and post-SIESTA, sleep-friendly orders rose in both units (foregoing vital signs: SIESTA unit, $4 \%$ to $34 \%$; standard, $3 \%$ to $22 \%, P<.001$ both; sleeppromoting VTE prophylaxis: SIESTA, $15 \%$ to $42 \%$; standard, $12 \%$ to $28 \%, P<.001$ both). In the SIESTA- enhanced unit, nighttime room entries dropped by $44 \%$ ( -6.3 disruptions/room, $P<.001)$, and patients were more likely to report no disruptions for nighttime vital signs $(70 \%$ vs $41 \%, P=.05)$ or medications $(84 \%$ vs $57 \%, P=.031$ ) than those in the standard unit. The standard unit was not changed. Although sleep-friendly orders were adopted in both units, a unit-based nursing empowerment approach was associated with fewer nighttime room entries and improved patient experience. Journal of Hospital Medicine 2019;14:38-41. (C) 2019 Society of Hospital Medicine

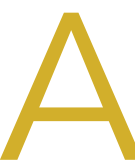

Ithough sleep is critical to patient recovery in the hospital, hospitalization is not restful, ${ }^{1,2}$ and inpatient sleep deprivation has been linked to poor health outcomes. ${ }^{1-4}$ The American Academy of Nursing's Choosing Wisely ${ }^{\circledR}$ campaign recommends nurses reduce unnecessary nocturnal care. ${ }^{5}$ However, interventions to improve inpatient sleep are not widely implemented. ${ }^{6}$ Targeting routine disruptions, such as overnight vital signs, by changing default settings in the electronic health record (EHR) with "nudges" could be a cost-effective strategy to improve inpatient sleep. ${ }^{4,7}$

We created Sleep for Inpatients: Empowering Staff to Act (SIESTA), which pairs nudges in the EHR with interprofessional education and empowerment, ${ }^{8}$ and tested its effectiveness on objectively and subjectively measured nocturnal sleep disruptors.

\section{METHODS}

\section{Study Design}

Two 18-room University of Chicago Medicine general-medicine units were used in this prospective study. The SIESTA-enhanced

*Corresponding Author: Vineet M. Arora, MD, MAPP, Email: varora@medicine. bsd.uchicago.edu; Telephone: 773-702-8157; Twitter: @futuredocs

Additional Supporting Information may be found in the online version of this article

Received: March 9, 2018; Revised: August 16, 2018; Accepted: August 29, 2018

(c) 2019 Society of Hospital Medicine DOI 10.12788/jhm.3091 unit underwent the full sleep intervention: nursing education and empowerment, physician education, and EHR changes. The standard unit did not receive nursing interventions but received all other forms of intervention. Because physicians simultaneously cared for patients on both units, all internal medicine residents and hospitalists received the same education. The study population included physicians, nurses, and awake English-speaking patients who were cognitively intact and admitted to these two units. The University of Chicago Institutional Review Board approved this study (12-1766; 16685B).

\section{Development of SIESTA}

To develop SIESTA, patients were surveyed, and focus groups of staff were conducted; overnight vitals, medications, and phlebotomy were identified as major barriers to patient sleep. ${ }^{9}$ We found that physicians did not know how to change the default vital signs order "every 4 hours" or how to batch-order morning phlebotomy at a time other than 4:00 AM. Nurses reported having to wake patients up at 1:00 Am for q8h subcutaneous heparin.

\section{Behavioral Nudges}

The SIESTA team worked with clinical informaticists to change the default orders in Epic ${ }^{\mathrm{TM}}$ (Epic Systems Corporation, 2017, Verona, Wisconsin) in September 2015 so that physicians would be asked, "Continue vital signs throughout the night?"10 Previously, this question was marked "Yes" by default and hidden. While the default protocol for heparin q8h was maintained, heparin q12h (9:00 AM and 9:00 PM) was introduced as an op- 
TABLE. Demographics of Patients $(N=1,083)$

\begin{tabular}{|c|c|c|c|c|}
\hline Characteristic & \multicolumn{2}{|c|}{ SIESTA-Enhanced Unit } & \multicolumn{2}{|c|}{ Standard Unit } \\
\hline $\begin{array}{l}\text { Gender } \\
\text { (\% female) }\end{array}$ & $58.6 \%$ & $60.1 \%$ & $57.9 \%$ & $54.6 \%$ \\
\hline $\begin{array}{l}\text { Length of Stay (days) } \\
\text { Median (IQR) }\end{array}$ & $4(2-7)$ & $5(2-8)$ & $4(2-8)$ & $5(3-8)$ \\
\hline Race (\% African- American) & $64.4 \%$ & $62.8 \%$ & $67.1 \%$ & $75.1 \%$ \\
\hline
\end{tabular}

\section{Sleep-Promoting Order Set Usage}

\begin{tabular}{lccc}
\hline $\begin{array}{l}\text { Vital Signs } \\
n=168 \text { uses }\end{array}$ & $11,6.5 \%$ & $104,62 \%$ & $7,4.2 \%$ \\
\hline $\begin{array}{l}\text { Heparin } \\
n=147 \text { uses }\end{array}$ & $23,15.6 \%$ & $73,49.7 \%$ & $16,10.9 \%$ \\
\hline $\begin{array}{l}\text { Patients Reporting a Sleep Disruption } \\
n=201 \text { surveyed }\end{array}$ & $48,59 \%$ & $11,34 \%$ & $23.8 \%$ \\
\hline
\end{tabular}

No major differences in demographics among patients admitted before and after SIESTA in each unit were observed. Although the difference is clinically small, patients admitted to the standard unit were older than those admitted to the SIESTA-enhanced unit in both periods $(P<.05)$.

tion, since $\mathrm{q} 12 \mathrm{~h}$ heparin is equally effective for VTE prophylaxis. ${ }^{11}$ Laboratory ordering was streamlined so that physicians could batch-order laboratory draws at 6:00 AM or 10:00 PM.

\section{SIESTA Physician Education}

We created a 20-minute presentation on the consequences and causes of in-hospital sleep deprivation and evidence-based behavioral modification. We distributed pocket cards describing the mnemonic SIESTA (Screen patients for sleep disorders, Instruct patients on sleep hygiene, Eliminate disruptions, Shut doors, Treat pain, and Alarm and noise control). Physicians were instructed to consider forgoing overnight vitals, using clinical judgment to identify stable patients, use a sleep-promoting VTE prophylaxis option, and order daily labs at 10:00 PM or 6:00 AM. An online educational module was sent to staff who missed live sessions due to days off.

\section{SIESTA-Enhanced Unit}

In the SIESTA-enhanced unit, nurses received education using pocket cards and were coached to collaborate with physicians to implement sleep-friendly orders. Customized signage depicting empowered nurses advocating for patients was posted near the huddle board. Because these nurses suggested adding SIESTA to the nurses' ongoing daily huddles at 4:00 PM and 3:00 AM, beginning on January 1, 2016, nurses were asked to identify at least two stable patients for sleep-friendly orders at the huddle. Night nurses incorporated SIESTA into their handoff to day nurses for eligible patients. Day nurses would then call physicians to advocate changing of orders.

\section{Data Collection Objectively Measured Sleep Disruptors}

Adoption of SIESTA orders from March 2015 to March 2016 was assessed with a monthly Epic ${ }^{\text {TM }}$ Clarity report. From August 1, 2015 to April 1, 2016, nocturnal room entries were recorded using the GOJO SMARTLINK ${ }^{\text {TM }}$ Hand Hygiene system (GOJO Industries Inc., 2017, Akron, Ohio). This system includes two components: the hand-sanitizer dispensers, which track dispenses (numerator), and door-mounted Activity Counters, which use heat sensors that react to body heat emitted by a person passing through the doorway (denominator for hand-hygiene compliance). For our analysis, we only used Activity Counter data, which count room entries and exits, regardless of whether sanitizer was dispensed.

\section{Patient-Reported Nighttime Sleep Disruptions}

From June 2015 to March 2016, research assistants administered a 10-item Potential Hospital Sleep Disruptions and Noises Questionnaire (PHSDNQ) to patients in both units. Responses to this questionnaire correlate with actigraphy-based sleep measurements., 92,13 Surveys were administered every other weekday to patients available to participate (eg, willing to participate, on the unit, awake). Survey data were stored on the REDCap Database (Version 6.14.0; Vanderbilt University, 2016, Nashville, Tennessee). Pre- and post-intervention Hospital Consumer Assessment of Healthcare Providers and Systems (HCAHPS) "top-box ratings" for percent quiet at night and percent pain well controlled were also compared. 


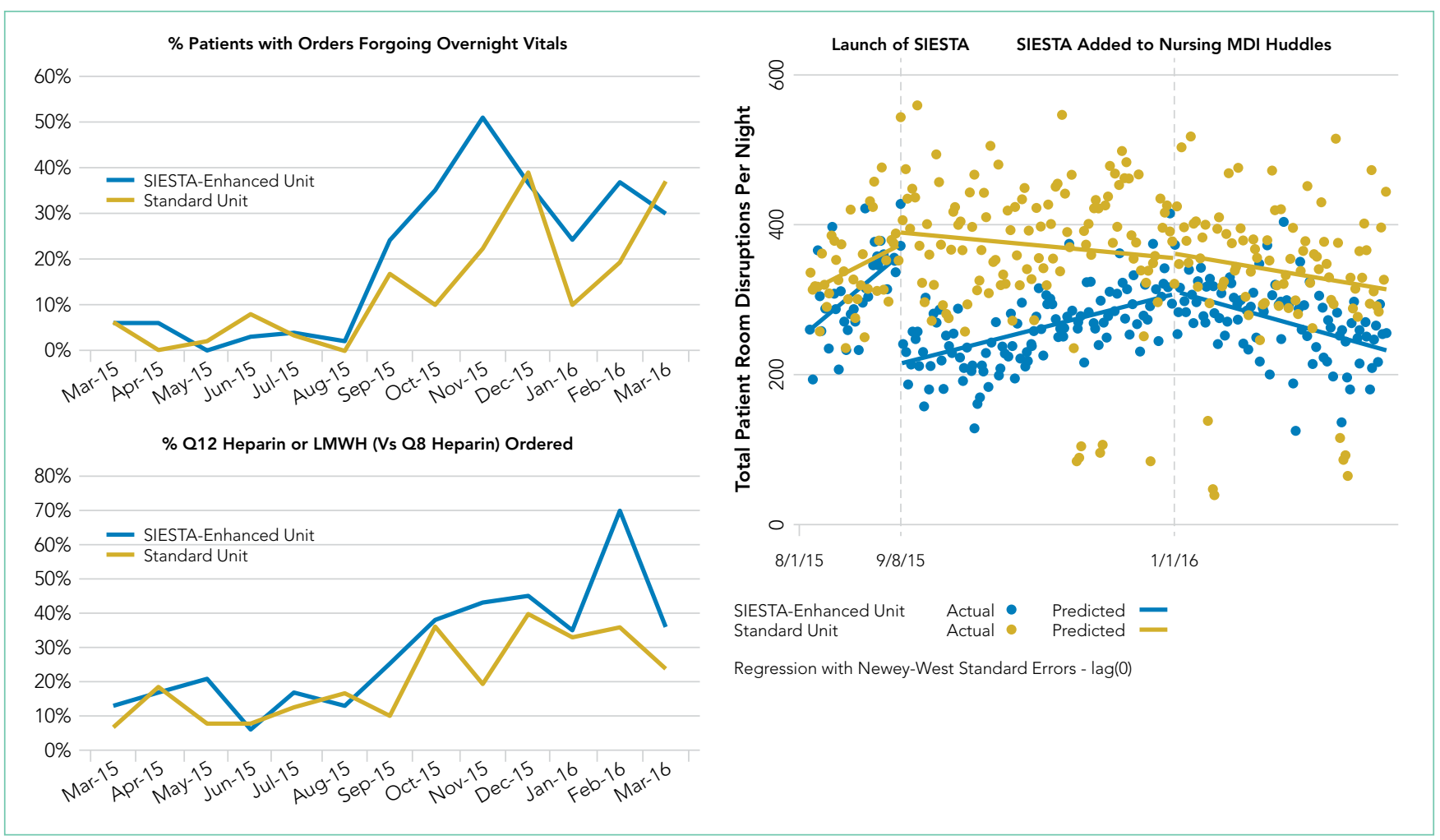

FIG. Sleep-promoting admission orders in EpicTM before and after SIESTA and interrupted time-series showing disruptions per night in SIESTA-enhanced and standard units.

Left: From admit orders with thromboembolism prophylaxis, the monthly percent of orders with $\mathrm{q}^{12 \mathrm{~h}}$ heparin or daily enoxaparin was calculated (vs q8h heparin). Patients who were not receiving for thromboembolism prophylaxis are excluded (ie, ongoing anticoagulation, bleeding, allergy, not indicated). From all admit orders per unit, the monthly percent of orders with discontinued overnight vitals was calculated.

Right: Using GOJO Activity Counters to measure entries into patient rooms over 244 nights, total disruptions per night were summed across the SIESTA-enhanced unit (18 patient rooms) and standard unit (18 patient rooms). Both the dotted and dashed lines are best-fit lines from regression models. In contrast to the standard unit (yellow), there were significant deceases in nocturnal room entries after the launch of SIESTA and implementation of the nursing huddle in the SIESTA-enhanced unit (blue).

\section{Data Analysis}

\section{Objectively Measured Potential Sleep Disruptors}

The proportion of sleep-friendly orders was analyzed using a two-sample test for proportions pre-post for the SIESTA-enhanced and standard units. The difference in use of SIESTA orders between units was analyzed via multivariable logistic regression, testing for independent associations between post-period, SIESTA-enhanced unit, and an interaction term (post-period $\times$ SIESTA unit) on use of sleep-friendly orders.

Room entries per night (11:00 PM-7:00 AM) were analyzed via single-group interrupted time-series. Multiple Activity Counter entries within three minutes were counted as a single room entry. In addition, the pre-post cutoff was set to 7:00 AM, September 8, 2015; after the SIESTA launch, a second cutoff marking when SIESTA was added to the nurses' MDI Huddle was added at 7:00 AM, January 1, 2016.

\section{Patient-Reported Nighttime Sleep Disruptions}

Per prior studies, we defined a score 2 or higher as "sleep disruption." ${ }^{\prime 9}$ Differences between units were evaluated via multivariable logistic regression to examine the association between the interaction of post-period $\times$ SIESTA-enhanced unit and odds of not reporting a sleep disruption. Significance was denoted as $P=.05$.

\section{RESULTS}

Between March 2015 and March 2016, 1,083 general-medicine patients were admitted to the SIESTA-enhanced and standard units (Table).

\section{Nocturnal Orders}

From March 2015 to March 2016, 1,669 Epic ${ }^{\text {TM }}$ general medicine orders were reviewed (Figure). In the SIESTA-enhanced unit, the mean percentage of sleep-friendly orders rose for both vital signs (+31\% [95\% Cl $\left.=25 \%, 36 \%] ; P<.001, \mathrm{n}_{\text {pre }}=306, \mathrm{n}_{\text {post }}=306\right]$ and VTE prophylaxis $\left(+28 \%[95 \% \mathrm{Cl}=18 \%, 37 \%] ; P<.001, \mathrm{n}_{\text {pre }}=158\right.$, $\left.n_{\text {post }}=173\right]$. Similar changes were observed in the standard unit for sleep-friendly vital signs (+20\% [95\% Cl = 14\%, 25\%]; $P<.001$, $\left.\mathrm{n}_{\text {pre }}=252, \mathrm{n}_{\text {post }}=219\right)$ and VTE prophylaxis $(+16 \%[95 \% \mathrm{Cl}=6 \%$, $\left.25 \%] ; P=.002, \mathrm{n}_{\text {pre }}=130, \mathrm{n}_{\text {post }}=125\right)$. Differences between the two units were not statistically significant, and no significant change in timing of laboratory orders postintervention was found.

\section{Nighttime Room Entries}

Immediately after SIESTA launch, an average decrease of 114 total entries/night were noted in the SIESTA-enhanced unit, ([95\% Cl $=-138,-91] ; P<.001)$, corresponding to a $44 \%$ reduction ( -6.3 entries/room) from the mean of 14.3 entries per patient room at baseline (Figure). No statistically significant 
change was seen in the standard unit. After SIESTA was incorporated into nursing huddles, total disruptions/night decreased by 1.31 disruptions/night $([95 \% \mathrm{Cl}=-1.64,-0.98]$; $P<$ .001) in the SIESTA-enhanced unit; by comparison, no significant changes were observed in the standard unit.

\section{Patient-Reported Nighttime Sleep Disruptions}

Between June 2015 and March 2016, 201 patient surveys were collected. A significant interaction was observed between the SIESTA-enhanced unit and post-period, and patients in the SIESTA-enhanced unit were more likely to report not being disrupted by medications (OR $4.08[95 \% \mathrm{Cl}=1.13-14.07] ; P=.031$ ) and vital signs (OR $3.35[95 \% \mathrm{Cl}=1.00-11.2]$; $P=.05$ ) than those in the standard unit. HCAHPS top-box scores for the SIESTA unit increased by 7\% for the "Quiet at night" category and 9\% for the "Pain well controlled" category; by comparison, no major changes $(>5 \%)$ were observed in the standard unit.

\section{DISCUSSION}

The present SIESTA intervention demonstrated that physician education coupled with EHR default changes are associated with a significant reduction in orders for overnight vital signs and medication administration in both units. However, addition of nursing education and empowerment in the SIESTA-enhanced unit was associated with fewer nocturnal room entries and improvements in patient-reported outcomes compared with those in the standard unit.

This study presents several implications for hospital initiatives aiming to improve patient sleep. ${ }^{14}$ Our study is consistent with other research highlighting the hypothesis that altering the default settings of EHR systems can influence physician behavior in a sustainable manner. ${ }^{15}$ However, our study also finds that, even when sleep-friendly orders are present, creating a sleep-friendly environment likely depends on the unit-based nurses championing the cause. While the initial decrease in nocturnal room entries post-SIESTA eventually faded, sustainable changes were observed only after SIESTA was added to nursing huddles, which illustrates the importance of using multiple methods to nudge staff.

Our study includes a number of limitations. It is not a randomized controlled trial, we cannot assume causality, and contamination was assumed, as residents and hospitalists worked in both units. Our single-site study may not be generalizable. Low HCAHPS response rates (10\%-20\%) also prevent demonstration of statistically significant differences. Finally, our convenience sampling strategy means not all inpatients were surveyed, and objective sleep duration was not measured.
In summary, at the University of Chicago, SIESTA could be associated with adoption of sleep-friendly vitals and medication orders, a decrease in nighttime room entries, and improved patient experience.

Disclosures: The authors have nothing to disclose.

Funding: This study was funded by the National Institute on Aging (NIA Grant No. T35AG029795) and the National Heart, Lung, and Blood Institute (NHLBI Grant Nos. R25HL116372 and K24HL136859).

\section{References}

1. Delaney LJ, Van Haren F, Lopez V. Sleeping on a problem: the impact of sleep disturbance on intensive care patients - a clinical review [published online ahead of print February 26, 2016]. Ann Intensive Care. 2015;5(3). doi: 10.1186/s13613-015-0043-2

2. Arora VM, Chang KL, Fazal $A Z$, et al. Objective sleep duration and quality in hospitalized older adults: associations with blood pressure and mood. J Am Geriatr Soc. 2011;59(11):2185-2186. doi: 10.1111/j.1532-5415.2011.03644.x.

3. Knutson KL, Spiegel K, Penev P, Van Cauter E. The metabolic consequences of sleep deprivation. Sleep Med Rev. 2007;11(3):163-178. doi: 10.1016/j. smrv.2007.01.002.

4. Manian FA, Manian CJ. Sleep quality in adult hospitalized patients with infection: an observational study. Am J Med Sci. 2015;349(1):56-60. doi: 10.1097/ MAJ.0000000000000355.

5. American Academy of Nursing announced engagement in National Choosing Wisely Campaign. Nurs Outlook. 2015;63(1):96-98. doi: 10.1016/j.outlook.2014.12.017.

6. Gathecha E, Rios R, Buenaver LF, Landis R, Howell E, Wright S. Pilot study aiming to support sleep quality and duration during hospitalizations. J Hosp Med. 2016;11(7):467-472. doi: 10.1002/jhm.2578.

7. Fillary J, Chaplin H, Jones G, Thompson A, Holme A, Wilson P. Noise at night in hospital general wards: a mapping of the literature. $\mathrm{Br} J$ Nurs. 2015;24(10):536-540. doi: 10.12968/bjon.2015.24.10.536.

8. Thaler R, Sunstein C. Nudge: Improving Decisions About Health, Wealth and Happiness. Yale University Press; 2008

9. Grossman MN, Anderson SL, Worku A, et al. Awakenings? Patient and hospital staff perceptions of nighttime disruptions and their effect on patient sleep. J Clin Sleep Med. 2017;13(2):301-306. doi: 10.5664/jcsm.6468.

10. Yoder JC, Yuen TC, Churpek MM, Arora VM, Edelson DP. A prospective study of nighttime vital sign monitoring frequency and risk of clinical deterioration. JAMA Intern Med. 2013;173(16):1554-1555. doi: 10.1001/jamainternmed.2013.7791.

11. Phung OJ, Kahn SR, Cook DJ, Murad MH. Dosing frequency of unfractionated heparin thromboprophylaxis: a meta-analysis. Chest. 2011;140(2):374-381. doi: 10.1378/chest.10-3084.

12. Gabor JY, Cooper AB, Hanly PJ. Sleep disruption in the intensive care unit. Curr Opin Crit Care. 2001;7(1):21-27

13. Topf M. Personal and environmental predictors of patient disturbance due to hospital noise. J Appl Psychol. 1985;70(1):22-28. doi: 10.1037/00219010.70.1.22.

14. Cho HJ, Wray CM, Maione S, et al. Right care in hospital medicine: co-creation of ten opportunities in overuse and underuse for improving value in hospital medicine. J Gen Intern Med. 2018;33(6):804-806. doi: 10.1007/ s11606-018-4371-4.

15. Halpern SD, Ubel PA, Asch DA. Harnessing the power of default options to improve health care. N Engl J Med. 2007;357(13):1340-1344. doi: 10.1056/ NEJMsb071595. 